\title{
Erratum: Effective field theory for double heavy baryons at strong coupling [Phys. Rev. D 102, 014013 (2020)]
}

\author{
Joan Soto and Jaume Tarrús Castellà®
}

(Received 10 August 2021; published 8 September 2021)

DOI: 10.1103/PhysRevD.104.059901

In Sec. IV when discussing the hyperfine splittings for the $l=2$ multiplets, off diagonal terms mixing $j=3 / 2,5 / 2$ with $\ell=3 / 2$ and $\ell=5 / 2$ were overlooked. To correct this omission the following changes should be made:

(a) The text from Eq. (42) to the end of Sec. 4 should be replaced by:

For $j=1 / 2$ and $7 / 2$ the contributions are

$$
\begin{aligned}
& M_{n \frac{1}{2} 2 \frac{3}{2}}^{(1)}=\frac{1}{2} \frac{\left\langle V_{(1 / 2)^{ \pm}}^{s 1}\right\rangle_{n 2}}{m_{Q}}-\frac{1}{3} \frac{\left\langle V_{(1 / 2)^{ \pm}}^{s 2}\right\rangle_{n 2}}{m_{Q}}-\frac{3}{2} \frac{\left\langle V_{(1 / 2)^{ \pm}}^{l}\right\rangle_{n 2}}{m_{Q}}, \\
& M_{n \frac{2}{2} 2 \frac{5}{2}}^{(1)}=\frac{1}{2} \frac{\left\langle V_{(1 / 2)^{ \pm}}^{s 1}\right\rangle_{n 2}}{m_{Q}}-\frac{2}{21} \frac{\left\langle V_{(1 / 2)^{ \pm}}^{s 2}\right\rangle_{n 2}}{m_{Q}}+\frac{\left\langle V_{(1 / 2)^{ \pm}}^{l}\right\rangle_{n 2}}{m_{Q}} .
\end{aligned}
$$

For $j=3 / 2,5 / 2$ we have the mixing matrices for $\ell=3 / 2$ and $\ell=5 / 2$ states

$$
\begin{aligned}
& M_{n_{2}^{2} 2}^{(1)}=\frac{1}{m_{Q}}\left(\begin{array}{cc}
\frac{1}{5}\left\langle V_{(1 / 2)^{ \pm}}^{s 1}\right\rangle_{n 2}-\frac{2}{15}\left\langle V_{(1 / 2)^{ \pm}}^{s 2}\right\rangle_{n 2}-\frac{3}{2}\left\langle V_{(1 / 2)^{ \pm}}^{l}\right\rangle_{n 2} & \frac{3}{5}\left\langle V_{(1 / 2)^{ \pm}}^{s 1}\right\rangle_{n 2}+\frac{1}{10}\left\langle V_{(1 / 2)^{ \pm}}^{s 2}\right\rangle_{n 2} \\
\frac{3}{5}\left\langle V_{(1 / 2)^{ \pm}}^{s 1}\right\rangle_{n 2}+\frac{1}{10}\left\langle V_{(1 / 2)^{ \pm}}^{s 2}\right\rangle_{n 2} & -\frac{7}{10}\left\langle V_{(1 / 2)^{ \pm}}^{s 1}\right\rangle_{n 2}+\frac{2}{15}\left\langle V_{(1 / 2)^{ \pm}}^{s 2}\right\rangle_{n 2}+\left\langle V_{(1 / 2)^{ \pm}}^{l}\right\rangle_{n 2}
\end{array}\right), \\
& M_{n_{2}^{52}}^{(1)}=\frac{1}{m_{Q}}\left(\begin{array}{cc}
-\frac{3}{10}\left\langle V_{(1 / 2)^{ \pm}}^{s 1}\right\rangle_{n 2}+\frac{1}{5}\left\langle V_{(1 / 2)^{ \pm}}^{s 2}\right\rangle_{n 2}-\frac{3}{2}\left\langle V_{(1 / 2)^{ \pm}}^{l}\right\rangle_{n 2} & \frac{\sqrt{14}}{5}\left\langle V_{(1 / 2)^{ \pm}}^{s 1}\right\rangle_{n 2}+\frac{1}{15} \sqrt{\frac{7}{2}}\left\langle V_{(1 / 2)^{ \pm}}^{s 2}\right\rangle_{n 2} \\
\frac{\sqrt{14}}{5}\left\langle V_{(1 / 2)^{ \pm}}^{s 1}\right\rangle_{n 2}+\frac{1}{15} \sqrt{\frac{7}{2}}\left\langle V_{(1 / 2)^{ \pm}}^{s 2}\right\rangle_{n 2} & -\frac{1}{5}\left\langle V_{(1 / 2)^{ \pm}}^{s 1}\right\rangle_{n 2}+\frac{4}{105}\left\langle V_{(1 / 2)^{ \pm}}^{s 2}\right\rangle_{n 2}+\left\langle V_{(1 / 2)^{ \pm}}^{l}\right\rangle_{n 2}
\end{array}\right) .
\end{aligned}
$$

We diagonalize to obtain the physical masses

$$
\begin{aligned}
M_{n_{2}^{2} 2 \pm}^{(1)}= & -\frac{1}{4 m_{Q}}\left\{\left\langle V_{(1 / 2)^{ \pm}}^{s 1}\right\rangle_{n 2}+\left\langle V_{(1 / 2)^{ \pm}}^{l}\right\rangle_{n 2} \pm \frac{1}{3}\left[81\left(\left\langle V_{(1 / 2)^{ \pm}}^{s 1}\right\rangle_{n 2}\right)^{2}+4\left(\left\langle V_{(1 / 2)^{ \pm}}^{s 2}\right\rangle_{n 2}\right)^{2}+225\left(\left\langle V_{(1 / 2)^{ \pm}}^{l}\right\rangle_{n 2}\right)^{2}\right.\right. \\
& \left.\left.-6\left\langle V_{(1 / 2)^{ \pm}}^{l}\right\rangle_{n 2}\left(27\left\langle V_{(1 / 2)^{ \pm}}^{s 1}\right\rangle_{n 2}-8\left\langle V_{(1 / 2)^{ \pm}}^{s 2}\right\rangle_{n 2}\right)\right]^{1 / 2}\right\}, \\
{ }_{M}^{\frac{5}{2} 2 \pm}(1)= & -\frac{1}{84 m_{Q}}\left\{21\left\langle V_{(1 / 2)^{ \pm}}^{s 1}\right\rangle_{n 2}-10\left\langle V_{(1 / 2)^{ \pm}}^{s 2}\right\rangle_{n 2}+21\left\langle V_{(1 / 2)^{ \pm}}^{l}\right\rangle_{n 2} \pm\left[3969\left(\left\langle V_{(1 / 2)^{ \pm}}^{s 1}\right\rangle_{n 2}\right)^{2}+156\left(\left\langle V_{(1 / 2)^{ \pm}}^{s 2}\right\rangle_{n 2}\right)^{2}\right.\right. \\
& +11025\left(\left\langle V_{(1 / 2)^{ \pm}}^{l}\right\rangle_{n 2}\right)^{2}+126\left\langle V_{(1 / 2)^{ \pm}}^{s 1}\right\rangle_{n 2}\left(10\left\langle V_{(1 / 2)^{ \pm}}^{s 2}\right\rangle_{n 2}+7\left\langle V_{(1 / 2)^{ \pm}}^{l}\right\rangle_{n 2}\right) \\
& \left.\left.-1428\left\langle V_{(1 / 2)^{ \pm}}^{s 2}\right\rangle_{n 2}\left\langle V_{(1 / 2)^{ \pm}}^{l}\right\rangle_{n 2}\right]^{1 / 2}\right\} .
\end{aligned}
$$

Let us consider the following hyperfine splittings among $l=2$ which are linear in the expectation values of the potentials

Published by the American Physical Society under the terms of the Creative Commons Attribution 4.0 International license. Further distribution of this work must maintain attribution to the author(s) and the published articles title, journal citation, and DOI. 


$$
\begin{aligned}
M_{n \frac{5}{2} 2+}+M_{n \frac{5}{2} 2-}-M_{n \frac{3}{2} 2+}-M_{n \frac{3}{2} 2-} & =\frac{5}{21 m_{Q}}\left\langle V_{(1 / 2)^{ \pm}}^{s 2}\right\rangle_{n 2}, \\
M_{n \frac{1}{2} 2 \frac{3}{2}}-\frac{1}{2}\left(M_{n \frac{3}{2} 2+}+M_{n \frac{3}{2} 2-}\right) & =\frac{1}{12 m_{Q}}\left(9\left\langle V_{(1 / 2)^{ \pm}}^{s 1}\right\rangle_{n 2}-4\left\langle V_{(1 / 2)^{ \pm}}^{s 2}\right\rangle_{n 2}-15\left\langle V_{(1 / 2)^{ \pm}}^{l}\right\rangle_{n 2}\right) \\
M_{n \frac{7}{2} 2 \frac{5}{2}}-\frac{1}{2}\left(M_{n \frac{3}{2} 2+}+M_{n \frac{3}{2} 2-}\right) & =\frac{1}{m_{Q}}\left(\frac{3}{4}\left\langle V_{(1 / 2)^{ \pm}}^{s 1}\right\rangle_{n 2}-\frac{2}{21}\left\langle V_{(1 / 2)^{ \pm}}^{s 2}\right\rangle_{n 2}+\frac{5}{4}\left\langle V_{(1 / 2)^{ \pm}}^{l}\right\rangle_{n 2}\right) .
\end{aligned}
$$

These formulas fix $\left\langle V_{(1 / 2)^{ \pm}}^{s 1}\right\rangle_{n 2},\left\langle V_{(1 / 2)^{ \pm}}^{s 2}\right\rangle_{n 2}$ and $\left\langle V_{(1 / 2)^{ \pm}}^{l}\right\rangle_{n 2}$ in terms of physical masses. Then, we have the following model-independent predictions

$$
\begin{aligned}
M_{n \frac{3}{2} 2+}^{(1)}-M_{n_{\frac{2}{2} 2-}}^{(1)}= & -\frac{1}{6 m_{Q}}\left[81\left(\left\langle V_{(1 / 2)^{ \pm}}^{s 1}\right\rangle_{n 2}\right)^{2}+4\left(\left\langle V_{(1 / 2)^{ \pm}}^{s 2}\right\rangle_{n 2}\right)^{2}+225\left(\left\langle V_{(1 / 2)^{ \pm}}^{l}\right\rangle_{n 2}\right)^{2}-6\left\langle V_{(1 / 2)^{ \pm}}^{l}\right\rangle_{n 2}\left(27\left\langle V_{(1 / 2)^{ \pm}}^{s 1}\right\rangle_{n 2}\right.\right. \\
& \left.\left.-8\left\langle V_{(1 / 2)^{ \pm}}^{s 2}\right\rangle_{n 2}\right)\right]^{1 / 2} \\
M_{n_{2} 2+}^{(1)}-M_{n_{2}^{5} 2-}^{(1)}= & -\frac{1}{42 m_{Q}}\left[3969\left(\left\langle V_{(1 / 2)^{ \pm}}^{s 1}\right\rangle_{n 2}\right)^{2}+156\left(\left\langle V_{(1 / 2)^{ \pm}}^{s 2}\right\rangle_{n 2}\right)^{2}+11025\left(\left\langle V_{(1 / 2)^{ \pm}}^{l}\right\rangle_{n 2}\right)^{2}+126\left\langle V_{(1 / 2)^{ \pm}}^{s 1}\right\rangle_{n 2}\right. \\
& \left.\times\left(10\left\langle V_{(1 / 2)^{ \pm}}^{s 2}\right\rangle_{n 2}+7\left\langle V_{(1 / 2)^{ \pm}}^{l}\right\rangle_{n 2}\right)-1428\left\langle V_{(1 / 2)^{ \pm}}^{s 2}\right\rangle_{n 2}\left\langle V_{(1 / 2)^{ \pm}}^{l}\right\rangle_{n 2}\right]^{1 / 2} .
\end{aligned}
$$

(b) In the third paragraph of Sec. $\mathrm{V}$ replace the following sentence:

"Both formulas turn out to be fulfilled extremely well, within $2 \mathrm{MeV}$."

by

"Both formulas are compatible with the lattice masses of Ref. [36]. Recall however that the uncertainties in the masses of the states involved are large."

(c) From Sec. VI (the Conclusions) remove the last sentence of the fifth paragraph:

"The data of Ref. [36] also allows us to check the relations in Eqs. (54) and (55) with agreement under 2 MeV."

Furthermore, the following misprints should also be corrected:

(1) In the second paragraph of the introduction "quark-antiquark duality" should be changed to "quark-diquark duality".

(2) In Eqs. (2), (3), and (4): $q^{l}(t, \boldsymbol{x}) \rightarrow q^{l}(t, \boldsymbol{R})$ and the $P_{+}$in the left-hand sides should be removed.

(3) In the text below Eq. (4) and above Eq. (5) $q_{\alpha}^{l}(t, \boldsymbol{x}) \rightarrow q_{\alpha}^{l}(t, \boldsymbol{R})$.

(4) In Table 2 the entry for $\eta_{P}$ for $\kappa^{p}=(1 / 2)^{ \pm}$and $l=3$ should read as $\mp$ instead of \pm .

(5) In the third line of Sec. 5: $M_{\Xi_{c}^{++}}^{*} \rightarrow M_{\Xi_{c c}^{*}}$.

(6) In Eq. (18) replace $\mathcal{O} \rightarrow \mathcal{Q}$, remove its dependence on $\boldsymbol{r}$, change the final dot for a comma and add following sentence after it:

"where $\mathcal{Q}_{\kappa^{p}}$ is the light-quark piece of the interpolating operator $\mathcal{O}_{\kappa^{p}}$."

(7) Below Eq. (18) replace " $r \gg \Lambda_{\mathrm{QCD}}$ " with " $r \gg 1 / \Lambda_{\mathrm{QCD}}$ ".

(8) In Eq. (19) replace " $\sigma_{Q Q q}$ " with " $\sigma$ ". 